\title{
OPEN Metabolic reprogramming of osteoclasts represents a therapeutic target during the treatment of osteoporosis
}

\author{
Jule Taubmann ${ }^{1,2}$, Brenda Krishnacoumar ${ }^{1,2}$, Christina Böhm ${ }^{1,2}$, Maria Faas ${ }^{1,2}$, \\ Dorothea I. H. Müller ${ }^{1,2}$, Susanne Adam ${ }^{1,2}$, Cornelia Stoll ${ }^{1,2}$, Martin Böttcher ${ }^{3}$, \\ Dimitrios Mougiakakos ${ }^{2,3}$, Uwe Sonnewald ${ }^{4}$, Jörg Hofmann ${ }^{4}$, Georg Schett ${ }^{1,2}$, \\ Gerhard Krönke $^{1,2 \rrbracket}$ \& Carina Scholtysek ${ }^{1,2 \bowtie}$
}

Osteoclasts are specialised bone resorbing cells that control both physiological and pathological bone turnover. Functional changes in the differentiation and activity of osteoclasts are accompanied by active metabolic reprogramming. However, the biological significance and the in vivo relevance of these events has remained unclear. Here we show that bone resorption of differentiated osteoclasts heavily relies on increased aerobic glycolysis and glycolysis-derived lactate production. While pharmacological inhibition of glycolysis did not affect osteoclast differentiation or viability, it efficiently blocked bone resorption in vitro and in vivo and consequently ameliorated ovariectomyinduced bone loss. Our experiments thus highlight the therapeutic potential of interfering with osteoclast-intrinsic metabolic pathways as possible strategy for the treatment of diseases characterized by accelerated bone loss.

Bone is a dynamic tissue undergoing constant remodeling that is orchestrated by osteoclast-mediated bone resorption and osteoblast-mediated bone formation. Osteoclasts (OCs) are formed from precursor cells (OCPs) of the monocyte/macrophage lineage in the presence of macrophage colony-stimulating factor (M-CSF) and receptor activator of NFKB ligand (RANKL) $)^{1,2}$. OCs are the primary bone-resorbing cells in both physiological and pathological states and thus play a key role in regulating bone mass. Pathologically enhanced osteoclast activity and accelerated bone resorption are observed during postmenopausal osteoporosis and diseases such as rheumatoid arthritis leading to decreased bone mass and increased risk of fractures ${ }^{3}$.

The molecular events underlying OC differentiation have been extensively studied. RANKL has been identified as key cytokine during osteoclastogenesis and its therapeutic targeting reduces osteoclast numbers and bone resorption during the treatment of osteoporosis. Mechanisms that control and adjust the function of mature osteoclasts are less well understood. However, insights into these pathways would be highly relevant to design novel therapeutic approaches to fine tune osteoclast function.

Recent data indicate that active metabolic reprogramming occurs during RANKL-induced osteoclastogenesis, where these multinucleated cells show an increase in mitochondrial content, biomass and mitochondrial respiration as well as accelerated glycolytic metabolism ${ }^{4-6}$. More recently, human osteoclasts were described to directly increase glycolysis during bone resorption in vitro. However, the in vivo consequences of these events remain unclear ${ }^{7}$. In this study, we used cell culture medium containing bone powder to induce a bone-resorbing phenotype in OCs and to study the consecutive functional and metabolic changes. We observed an active metabolic switch in bone resorbing OCs, characterized by increased glycolysis and lactate production. Notably, both

\footnotetext{
${ }^{1}$ Department of Internal Medicine 3, Friedrich Alexander University of Erlangen-Nuremberg (FAU) and Universitätsklinikum Erlangen, Erlangen, Germany. ${ }^{2}$ Deutsches Zentrum für Immuntherapie (DZI), University of Erlangen-Nuremberg and Universitätsklinikum Erlangen, Erlangen, Germany. ${ }^{3}$ Department of Internal Medicine 5, University of Erlangen-Nuremberg and Universitätsklinikum Erlangen, Erlangen, Germany. ${ }^{4}$ Division of Biochemistry, Department of Biology, University of Erlangen-Nuremberg, Erlangen, Germany. ${ }^{\square}$ email: gerhard.kroenke@uk-erlangen.de; carina.scholtysek@uk-erlangen.de
} 
A

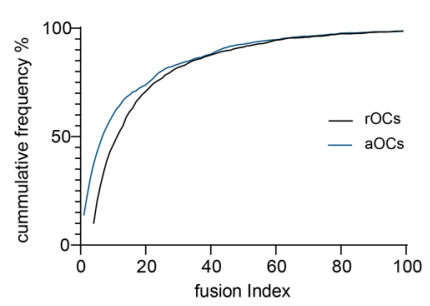

C

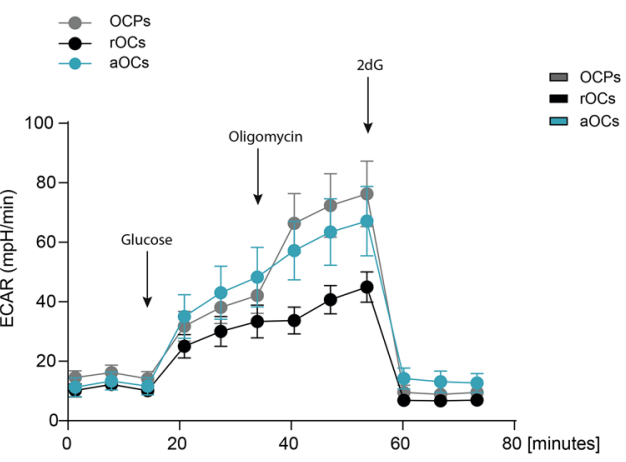

D

- OCPs
- rOCs
- aOCs

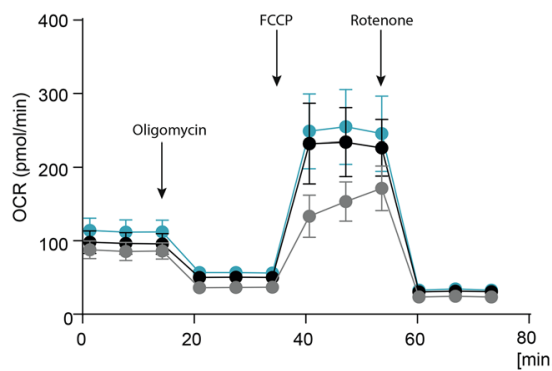

E

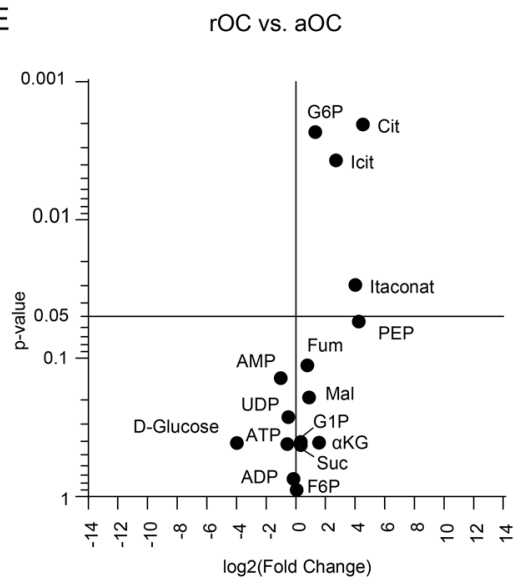

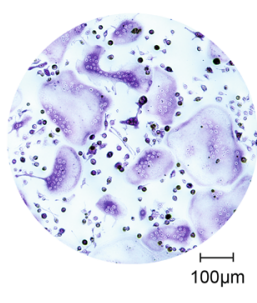

믈
rOCs
aOCs
OCPs
rOCs
aOCs

$\mathrm{F}$ 므으s
ㅁocs
$\square$ aOCs

G

므s
rOCs
口 aOCs
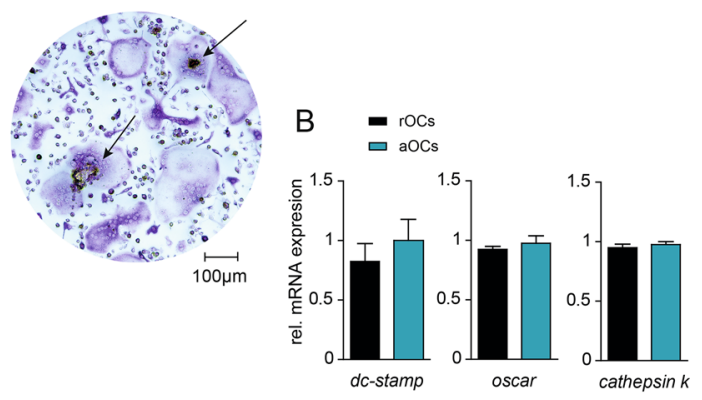
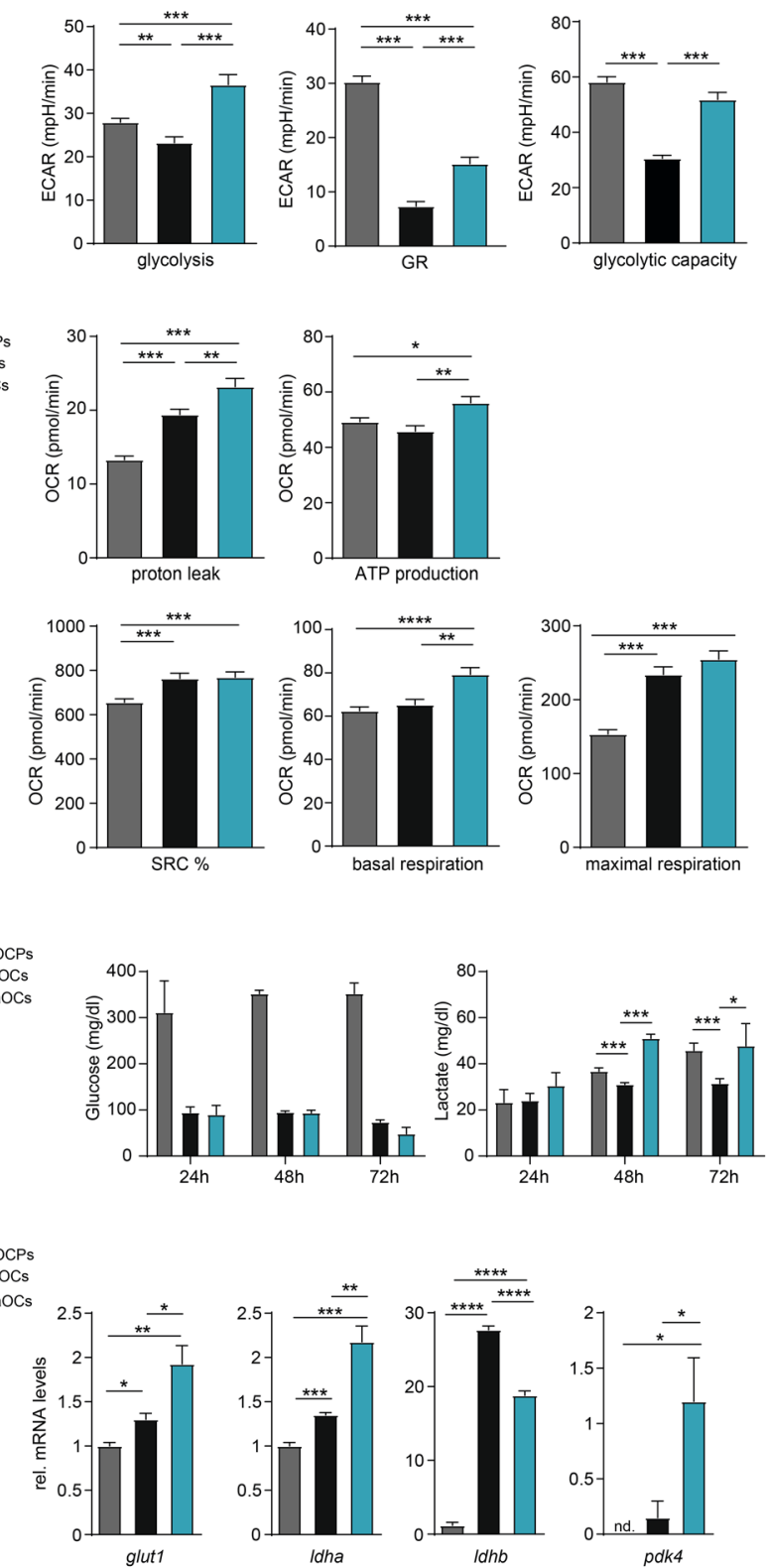
4Figure 1. Activation of osteoclasts is paralleled by increased glycolysis. (A) Fusion index (left panel) and representative TRAP staining (right panel) of OCPs, rOCs and aOCs on day 4 of osteoclast cell culture. (B) Quantitative RT-PCR measuring relative mRNA levels of dc-stamp, oscar and cathepsin $k$, normalized on beta-actin after day 3 of osteoclast cell culture in rOCs and aOCs. (C) Extracellular acidification rate (ECAR) including glycolysis, glycolytic capacity and glycolytic reserve (GR), measured with an extracellular flux (XF) analyzer in rOCs and aOCs after $48 \mathrm{~h}$. (D) Oxygen consumption rate (OCR) including basal and maximal respiration rate, ATP production, proton leak and spare respiratory capacity (SRC), measured with an extracellular flux (XF) analyzer in OCPs, rOCs and aOCs after 48 h. (E) Mass spectrometry-based analysis of the abundance of indicated metabolites in rOCs and aOCs (F) Glucose consumption and lactate accumulation in culture media at indicated time points of OC cell culture comparing OCPs, rOCs and aOCs. (G) Quantitative RT-PCR determining relative mRNA levels of glucose transporter 1 (glut1), Lactate dehydrogenase A (ldha), Lactate dehydrogenase B $(l d h b)$ and Pyruvate dehydrogenase lipoamide kinase isozyme $4(p d k 4)$, normalized on beta-actin after indicated time points of OC cell culture.

glycolysis and its metabolite lactate were essential for efficient bone resorption. Functional inhibition of glycolysis by 2-Desoxy-D-glucose (2-DG) blocked bone resorption by OCs, which could be rescued by supplementation of either pyruvate or lactate suggesting lactate as an active metabolic intermediate that drives bone resorption. In accordance with a key role of glycolysis during OC-mediated bone resorption in vivo, we observed effective amelioration of ovariectomy-induced bone loss in mice that received 2-DG or a small molecular inhibitor of lactate dehydrogenase A (LDH-A).

\section{Results}

Activation of osteoclasts is paralleled by increased aerobic glycolysis. To analyse changes in the metabolic activity of bone resorbing OCs, we isolated murine bone marrow-derived monocytes as OCPs and cultured these cells in M-CSF and RANKL containing medium in the absence and presence of bone powder to simulate a bone resorbing metabolic state in such "activated" OCs (aOCs). The application of bone powder medium did not affect OC fusion capacity, OC number or the expression of OC differentiation marker genes, such as dc-stamp, oscar and cathepsin $k$ (Fig. 1A,B). By performing extracellular flux assays, we compared changes in the extracellular acidification rate (ECAR) as measure of the glycolytic activity between OPCs, nonactivated "resting" OCs (rOCs) and bone resorbing aOCs. OC differentiation resulted in a reduced glycolytic activity in rOCs in comparison to OCPs (Fig. 1C). However, aOCs displayed increased glycolysis, glycolytic reserve (GR) and glycolytic capacity when compared to rOCs, confirming that bone resorption was paralleled by accelerated extracellular acidification and an enhanced aerobic glycolysis (Fig. 1C). Both rOCs and aOCs populations revealed marked elevation of their oxygen consumption rate (OCR), indicated by increased spare respiratory capacity (SRC) and maximal respiration. Moreover, rOCs and aOCs showed an elevated proton leak and basal respiration demonstrating increased mitochondrial respiration during OC differentiation and bone resorption (Fig. 1D).

In addition, we performed metabolic profiling of rOCs and aOCs. In accordance with the data derived from the extracellular flux assays, we observed an increase of metabolites of both glycolysis and the Krebs cycle such as glucose 6-phosphate (G6P) or citrate (Cit), respectively, upon initiation of bone resorption in aOCs (Fig. 1E). Direct measurement of glucose consumption and lactate accumulation in the growth media of rOCs and aOCs revealed that both OC populations substantially consumed glucose. However, lactate production was only significantly elevated in bone-resorbing aOCs, which was in accordance with their enhanced state of aerobic glycolysis (Fig. 1F). When we analysed the metabolic gene expression profile, we found several genes of the glycolytic pathway induced in aOCs (Fig. 1G). In particular, expression of glut 1 , ldha and pdk4 were increased and provided an explanation for the increase in the rate of glycolysis and enhanced lactate production. On the contrary, $l d h b$, which catalyses the conversion of lactate and NAD + to pyruvate, was downregulated in aOCs, indicating a metabolic shift towards active aerobic glycolysis and lactate production during bone resorption.

Glycolysis-derived lactate essentially supports bone resorption. Next, we aimed to address the functional consequences of the observed metabolic reprogramming of aOCs including the increase in aerobic glycolysis and lactate production on osteoclast differentiation and bone resorption. We therefore isolated monocytes, assessed OC differentiation and quantified their bone resorbing capacity in the presence of specific inhibitors of oxidative phosphorylation and glycolysis, respectively. Notably, 2dG-mediated inhibition of glycolysis efficiently blocked bone resorption, but did not affect OC differentiation and fusion, whereas rotenone as an inhibitor of mitochondrial complex I, decreased osteoclastogenesis but did only moderately affect resorption activity (Fig. 2A,B). These results indicated that aerobic glycolysis in OCs was essential for OC activation and bone resorption, but did not affect earlier steps of osteoclastogenesis such as differentiation and fusion events. To determine downstream mediators and metabolites that were responsible for the glycolysis-mediated increase in bone resorption, we assessed the impact of the glycolysis products lactate and pyruvate on the bone resorption capacity of aOCs in the absence and presence of $2 \mathrm{dG}$. While lactate and pyruvate alone did not affect the bone resorbing activity of aOCs, both metabolites rescued the decreased bone resorption caused by inhibition of glycolysis via $2 \mathrm{dG}$ (Fig. $2 \mathrm{C}$ ), which suggested lactate as the active metabolite mediating bone resorption in response to enhanced glycolysis in aOCs.

Inhibition of glycolysis and lactate production ameliorate ovariectomy-induced bone loss. To assess the potential of OC-mediated glycolysis as therapeutic target for diseases characterized by a pathologi- 
A

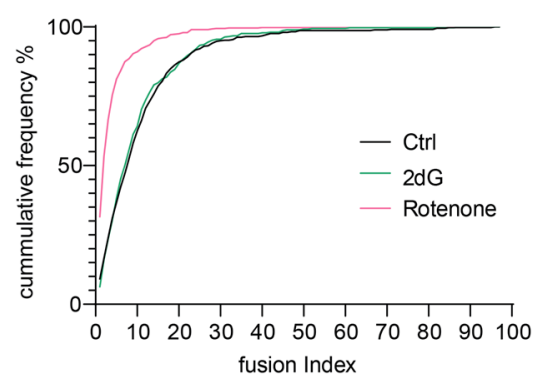

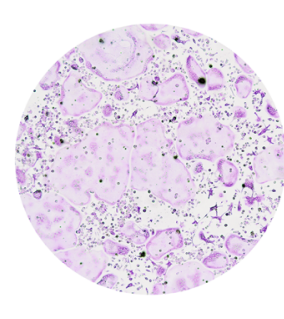

control

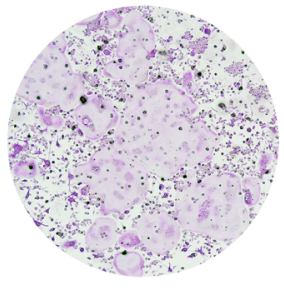

$2 d G$

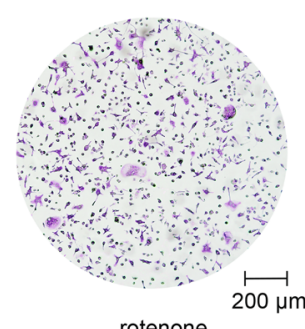

rotenone

$\mathrm{B}$

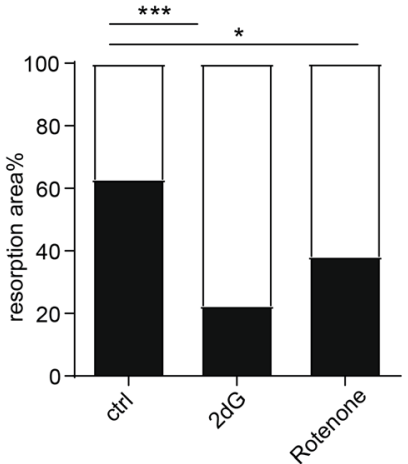

resorbed area bone

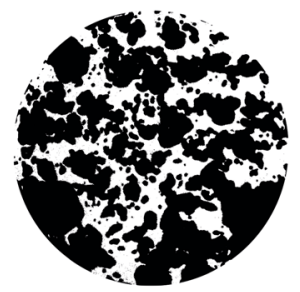

control

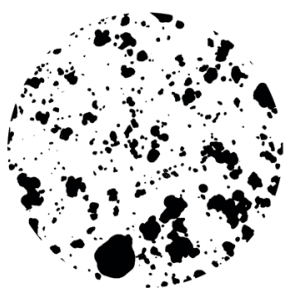

$2 d G$

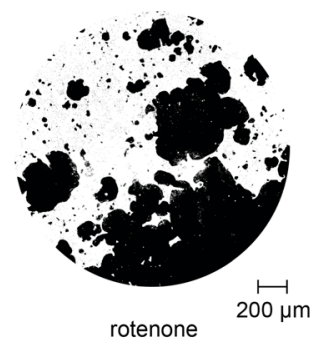

C $\square$ resorbed area
$\square$ bone

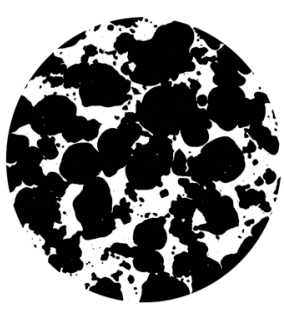

control

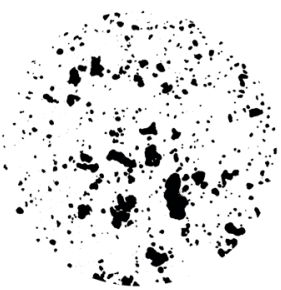

$2 d G$

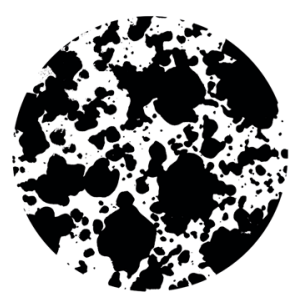

lactate

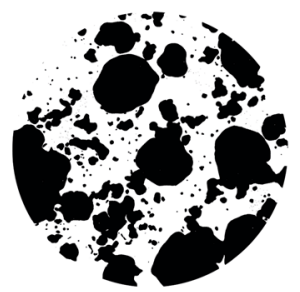

$2 d G+$ lactate
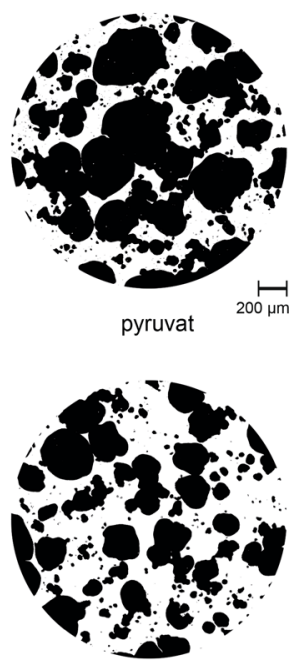

$2 d G+$ pyruvat

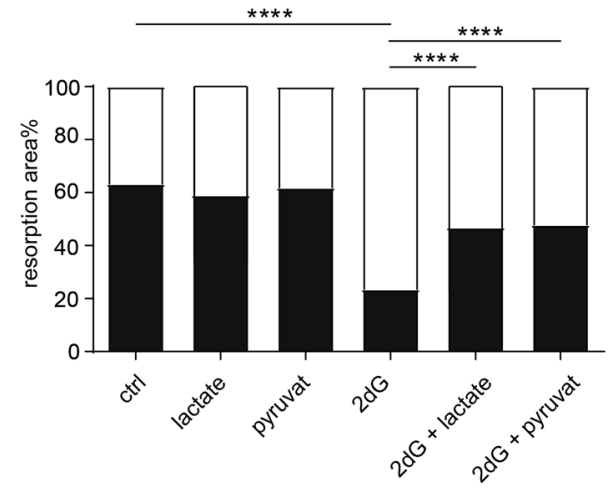

Figure 2. Glycolysis routes bone resorptive activity in vitro. (A) Fusion index (left) and representative TRAP staining of OCs differentiated in the absence or presence of the glycolysis inhibitor 2-Deoxy-d-glucose (2dG; $10 \mu \mathrm{M})$ or rotenone $(0.02 \mu \mathrm{M})$ on day 3 of OC culture. (B,C) OCs were generated on bone resorption plates for 5 days and stimulated with $2 \mathrm{dG}(10 \mu \mathrm{M})$ or rotenone $(0.02 \mu \mathrm{M})$ in the presence or absence of lactate $(5 \mathrm{mM})$ or pyruvate $(2 \mathrm{mM})$ as indicated. The percentage of the resorbed area was quantified via Photoshop and pictures show representative images of resorption pit formation.

cally enhanced bone resorption, we decided to study the effect of blocking glycolysis and lactate production during a mouse model of ovariectomy-induced bone loss. Therefore, we treated mice that displayed an established osteoporosis (treatment start 2 weeks post ovariectomy) with the glycolysis inhibitor $2 \mathrm{dG}$ for 6 additional weeks before bone structure was analysed by $\mu \mathrm{CT}$ (Fig. $3 \mathrm{~A}$ ). $2 \mathrm{dG}$ treatment was well tolerated. As expected, mice that had undergone ovariectomy displayed an increased weight gain over the study period of 8 weeks, which was slightly decreased by treatment with $2 \mathrm{dG}$ (Fig. 3B). Analysis of the trabecular microstructure of the spine showed that $2 \mathrm{dG}$ slightly decreased bone density in the sham-operated group, whereas we observed an increase in bone density upon $2 \mathrm{dG}$ treatment in mice that had undergone ovariectomy (Fig. 3C,D). 2dG treatment resulted in an increased bone volume/total volume (BV/TV), an increased bone mineral density (BMD) 
and an increased trabecular thickness in ovariectomized mice, which was in accordance with a block of pathologic bone resorption in these mice.

We additionally validated these results and our in vitro findings in a second round of experiments where we treated mice with the LDHA inhibitor GSK2837808A to selectively interfere with lactate production in vivo. GSK2837808A inhibited the bone resorbing activity of osteoclasts to a similar extend as 2dG without cytotoxic effects in vitro (Suppl. Fig. 1A,B). Treatment was started 2 weeks after ovariectomy in mice that already had developed bone loss (Fig. 3E). In accordance with our previous results, block of lactate synthesis resulted in a slight decrease in bone density in sham- operated mice, whereas GSK2837808A treatment resulted in a significant improvement of ovariectomy-induced bone loss (Fig. 3F,G). Here, we observed an increased BV/TV, an increased trabecular thickness as well as an increased BMD in ovariectomized mice that had received GSK2837808A.

\section{Discussion}

Increasing data highlight the mutual influence of cellular metabolic processes and the functional properties of innate and adaptive immune cells. Among others, macrophages were shown to undergo a defined metabolic reprogramming in response to different activation stimuli. Pro-inflammatory classically-activated macrophages e.g. increase their glycolytic activity and shut down their mitochondrial respiration, whereas anti-inflammatory alternatively-activated macrophages show an increase in mitochondrial respiration. These contrasting metabolic adaptions of individual macrophage subsets seem to support distinct activation and differentiation programs, thereby promoting either onset or resolution of inflammation ${ }^{8}$. Our current data confirm that osteoclast, as mononuclear phagocyte-derived cells that settle the bone microenvironment, undergo specific metabolic adaptions during differentiation and bone resorption as well.

Notably, and in contrast to many other immune cells, bone resorbing and activated osteoclasts are not characterized by an isolated, but by a simultaneous increase of both glycolytic activity and mitochondrial respiration when compared to resting osteoclasts. This observation suggests that the process of bone resorption substantially increases the metabolic and energetic requirements of osteoclasts. This adaptation might be merely linked to an increased demand in energy. Alternatively, it points to the necessity of a provision of specific and essential metabolites that derive from glycolysis and/or mitochondrial respiration and support the functional properties of osteoclasts during bone resorption. Our data primarily support the latter scenario and suggest that glycolysisderived lactate is essential for the efficient resorption of bone by osteoclasts. Block of oxidative phosphorylation, in turn, only moderately affected bone resorption, although osteoclast differentiation was clearly impaired. Block of glycolysis by $2 \mathrm{dG}$ or block of the conversion of pyruvate to lactate by the LDHA-inhibitor GSK2837808A accordingly interfered with regular bone resorption, which could be rescued by the restoration of regular lactate levels. The exact role of lactate during this process remains unclear at the moment. Our in vitro experiments show a substantial increase in extracellular lactate upon initiation of bone resorption. A likely possibility is therefore a role of lactate during the acidification of the osteoclast resorption pit, which is an essential step during bone resorption by osteoclasts, facilitates the activation of acid proteases that brake down bone matrix, and additionally dissolves bone minerals.

Our data additionally show that blocking glycolysis and lactate production represents efficient strategies to interfere with pathological osteoclast-mediated bone loss during ovariectomy as a model of postmenopausal osteoporosis that is usually characterized by increased differentiation and activation of osteoclasts ${ }^{9}$. Although such a strategy significantly increased bone mass in osteoporotic mice, healthy mice displayed a slightly decreased bone density upon block of glycolysis. Although the underlying reasons remain to be elucidated, block of glycolysis likely affects other cell types such as osteoblasts and osteocytes that are equally relevant for a regular bone homeostasis. Especially osteoblast are dependent on glycolysis to form new bone ${ }^{10}$, although they equally require mitochondrial respiration and fatty acids to mineralize newly formed extracellular matrix ${ }^{11}$.

Taken together, our data identify aerobic glycolysis and osteoclast-mediated lactate production as essential steps during bone resorption and suggest that blocking glycolysis and lactate production in osteoclasts represents a potential therapeutic strategy during diseases that are characterised by accelerated osteoclast-mediated bone loss such as postmenopausal osteoporosis and rheumatoid arthritis.

\section{Methods}

The authors confirm that all methods were carried out in accordance with relevant guidelines and regulations. The ethical approval for all animal experiments was carried out in accordance with relevant guidelines and regulations by the ethical committee of the Friedrich Alexander University of Erlangen-Nuremberg (FAU), Germany.

Mice. Mice were maintained at the specific pathogen-free animal care facility (FPZ) of the University of Erlangen-Nuremberg and housed in a room at $23 \pm 2{ }^{\circ} \mathrm{C}$, with $50 \pm 10 \%$ humidity and a 12-h light/dark cycle (lights on from 08:00 a.m. to 08:00 p.m.). All mice were allowed free access to water and regular rodent chow.

Ovariectomy-inducd osteoporosis (OVX). Female C57BL/6JRj wild-type mice were obtained from Janvier Labs and experiments were performed according to guidelines of laboratory animal care and use. All efforts were made to reduce the number of animals tested and their suffering. For ovariectomy, mice (12 weeks of age) were anaesthetized with a ketamine-xylazine (KX) solution and bilaterally ovariectomized, while ovaries of the sham group were left intact. The ovariectomized mice were allowed to recover for two weeks to ensure the development of osteoporosis. After 6 full weeks of treatment with vehicle, 2d or GSK 2837808A, all animals were killed via cervical dislocation under $\mathrm{CO}_{2}$ anaesthesia and one tibia was excised for histological analyses and one for microcomputer tomography $(\mu \mathrm{CT})$ imaging. Successful ovariectomy was examined at the day of preparation by anatomical analysis of the ovaries. 
A

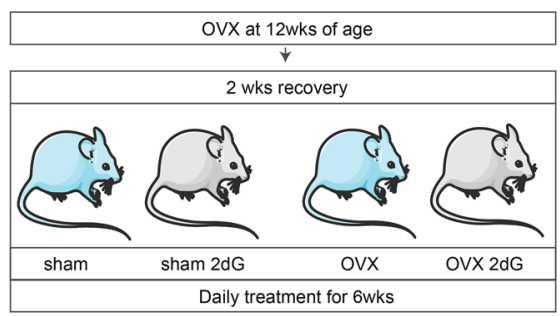

B

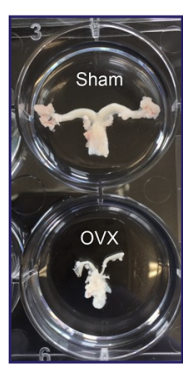

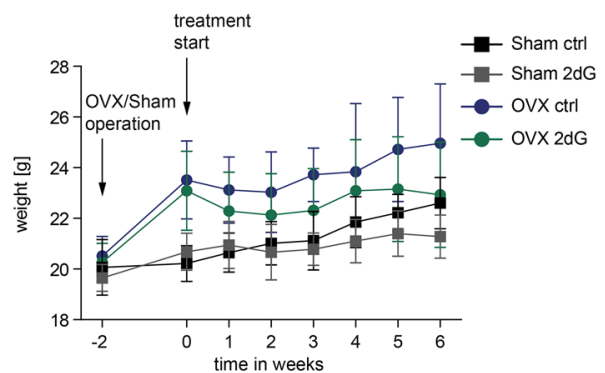

C

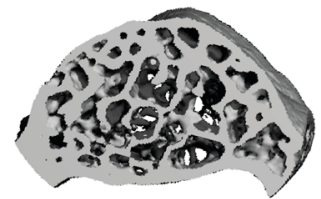

Sham

D
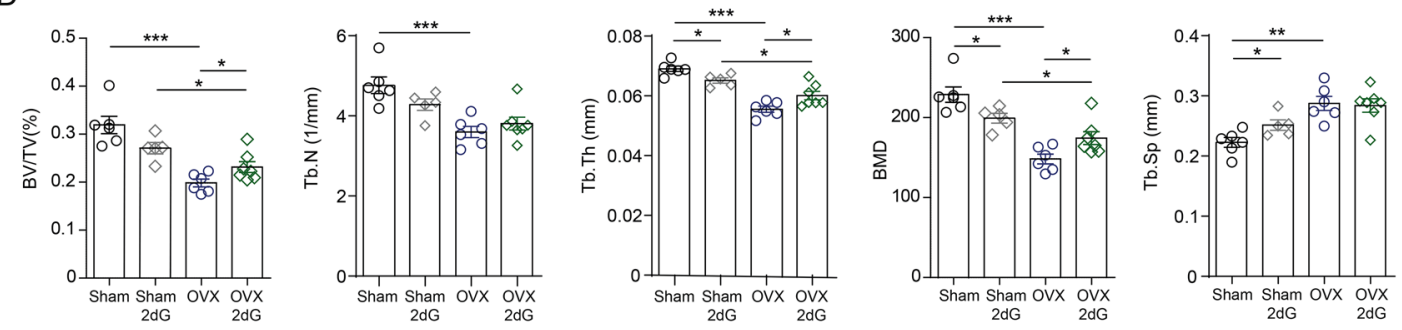

E

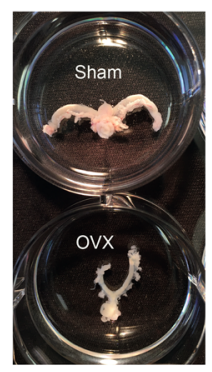

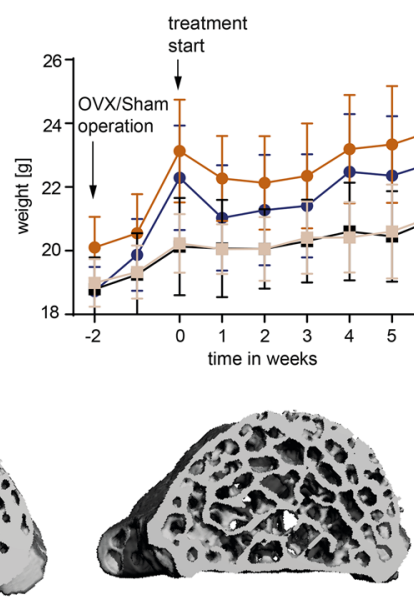

Sham
GSK

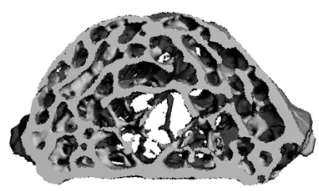

ovx

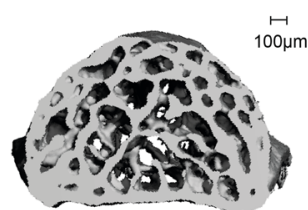

$O V X$
$2 d G$
F

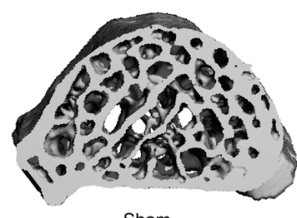

Sham

G

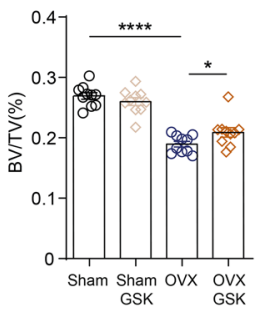

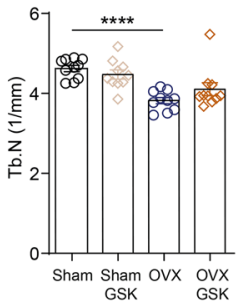

$\rightarrow$ Sham ctrl

- Sham GSK

$\rightarrow$ ovX ctrl

$\rightarrow$ OVXGSK 
४Figure 3. Inhibition of glycolysis and lactate production ameliorates OVX-induced bone loss. (A) Schematic diagram illustrating the postmenopausal mouse model of ovariectomy-induced bone loss and treatment strategies. (B) Weight course in ovariectomized (OVX) or sham-operated mice after treatment with $2 \mathrm{dG}$ treatment or treatment with a vehicle (aqua injectabile), respectively. (C) Representative images show spinal architecture by $\mu \mathrm{CT}$ reconstruction. (D) $\mu \mathrm{CT}$ measurements of indicated parameters of spinal microarchitecture including bone volume to total volume (BV/TV), trabecular number (Tb.N.), trabecular thickness (Tb.Th.) and trabecular separation (Tb.Sp.) in ovariectomized (OVX) or sham-operated mice after treatment with $2 \mathrm{dG}$, respectively. (E) Demonstration of adnexe from Sham mouse and ovariectomized mouse 8 weeks after surgery and course of weight gain in ovariectomized (OVX) or sham-operated mice after treatment with the LDHA inhibitor GSK2837808A (GSK) or a vehicle (DMSO). (F) Representative images show spinal architecture of indicated groups by $\mu \mathrm{CT}$ reconstruction. (G) $\mu \mathrm{CT}$ measurements of indicated parameters of spinal microarchitecture including bone volume to total volume (BV/TV), trabecular number (Tb.N). trabecular thickness (Tb.Th.) and trabecular separation (Tb.Sp) in ovariectomized (OVX) or sham-operated mice after treatment with GSK or the vehicle.

Treatment with 2dG. Mice were divided into four groups: sham operated mice (sham, $\mathrm{n}=6$ ), ovariectomized mice (OVX, $n=6), 2 \mathrm{dG}$ treated OVX mice $(\mathrm{OVX}+2 \mathrm{dG}, \mathrm{n}=7)$ and $2 \mathrm{dG}$ treated sham operated mice $(\mathrm{sham}+2 \mathrm{dG}, \mathrm{n}=5)$. Mice were treated by intra-peritoneal injections with 2 -Desoxy-Glucose $(500 \mathrm{mg} / \mathrm{kg} / \mathrm{d}$ body weight, suspended in aqua injectabile) daily for 6 weeks. Control groups were treated with a vehicle (aqu. inj.).

Treatment with GSK 2837808A. Mice were treated by oral gavage with LDH-A inhibitor GSK 2837808A $(8 \mathrm{mg} / \mathrm{kg} / \mathrm{d}$ body weight, suspended in vehicle of $3 \%$ DMSO and $1 \%$ methylcellulose; Tocris Cat. No. 5189) daily for 6 weeks. Control groups were treated with vehicle (3\% DMSO).

Microcomputer tomography imaging $(\mu \mathrm{CT})$. Bones were fixed in $4 \%$ paraformaldehyde overnight prior to the analyses. All $\mu \mathrm{CT}$ imaging was performed using the cone-beam Desktop Micro Computer Tomograph “ $\mu$ CT 40 ” by SCANCO Medical AG, Bruettisellen, Switzerland (http://www.scanco.ch/en/systems-solut ions/specimen/microct $40 . \mathrm{html}$ ). The settings were optimized for calcified tissue visualization at $55 \mathrm{kVp}$ with a current of $145 \mu \mathrm{A}$ and $200 \mathrm{~ms}$ integration time for 500 projections $/ 180^{\circ}$. For the segmentation of $3 \mathrm{D}$-Volumes an isotropic voxel size of $8,4 \mu \mathrm{m}$ and an evaluation script with adjusted greyscale thresholds of the operating system "Open VMS" by SCANCO Medical was used. For 3D- $\mu$ CT analysis, measurement was conducted $1680 \mu \mathrm{m}$ ca. $400 \mu \mathrm{m}$ below the approximate middle of growth plate, left or right tibia metaphysis.

Cell culture. Bone marrow derived monocytes (BMDMs) were isolated as previously described and differentiated into osteoclast $(\mathrm{OCs})^{8}$. Briefly, hematopoietic bone marrow cells were purified from tibial bone with a $70-\mu \mathrm{m}$ cell strainer, cultured overnight and stimulated with appropriate growth medium (MEM Alpha Medium $1 \times$ GlutaMAX, GIBCO; \#32571-028, containing 10\% L929 conditioned medium, 10\% FCS and 1\% penicillin/streptomycin) at $37^{\circ} \mathrm{C}$ with $5 \% \mathrm{CO}_{2}$. OCPs were cultured in OC-growth medium (MEM Alpha Medium $1 \times$ GlutaMAX, GIBCO; \#32571-028, containing 10\% L929 conditioned medium, $10 \mathrm{ng} / \mathrm{ml}$ RANKL, 10\% FCS and $1 \%$ penicillin/streptomycin) for $24 \mathrm{~h}$. Osteoclast differentiation was quantified by counting multinucleated (>3 nuclei) TRAP + cells on day 4 of OC culture using the Acid Phosphatase, Leukocyte (TRAP) Kit (SigmaAldrich, \# 387A). Osteoclast fusion events were quantified by counting the nuclei per osteoclast.

Calculation of the fusion index. For analysis of the fusion efficiency, a fusion index calculated. The total number of cell fusion events in one syncytium (number of nuclei/osteoclast) were counted (= fusion index). Multinucleated osteoclasts were defined as $>3$ nuclei/cell. The absolute number of cells with the same amount of nuclei were set in ratio with the total number of cells per setting and given in cumulative frequency in percent in order to define the fusion efficiency.

Bone resorption analysis. For analysis of osteoclast resorption, osteoclast differentiation was conducted in Osteoplates (Corning Osteo Assay Surface 24-well Multiple Well Plates; \#3987). Experiments were stopped by aspirating the medium and adding $10 \%$ bleach solution for $5 \mathrm{~min}$ at room temperature. Afterwards, bleach solution was aspirated and cells were washed twice with $150 \mu \mathrm{l}$ of $\mathrm{dH} 2 \mathrm{O}$ and air-dried overnight. Pictures were taken with Zeiss Axioskop 2 microskop ( $5 \times$ enlargement) and converted into black and white with Adobe Photoshop. Osteoclast resorption activity was quantified by measurement of black (= resorption area) versus white (= bone) pixels with a Histogram.

Bone powder medium. Bovine bone meal box bone powder (from "Hund und Sport Hungenberg"; \#KNM3) was crushed into fine fragments with sterile backed mortar. Bone powder was stored in the freezer. Needed concentrations were measured and filled up with not heat inactivated FCS. Tubes were heat inactivated in a water bath for $1 \mathrm{~h}$ at $56^{\circ} \mathrm{C}$. One sample was separated on two bacterial dishes and plated under UV light overnight. Finally $5 \mathrm{ml}$ of heat inactivated and sterilized FCS containing Bone Powder was filled up with $45 \mathrm{ml}$ of growth medium (MEM Alpha Medium 1× GlutaMAX, GIBCO; \#32571-028, containing 10\% L929 conditioned medium, $10 \%$ FCS and $1 \%$ penicillin/streptomycin) to obtain bone powder medium. 
RT-PCR analysis. Total RNA was isolated from cells using peqGOLD TRIFast (peqlab, Germany). $1 \mu \mathrm{g}$ was used for the first-strand complementary DNA synthesis (Amersham Biosciences), which was then used for SYBR Green-based quantitative RT-PCR as described previously ${ }^{12}$. Triplicates were performed according to the manufacturer's instructions. The following mouse RT-PCR primer sequences were used:

\begin{tabular}{|l|l|l|}
\hline Primer & Forward sequence & Reverse sequence \\
\hline beta-actin & TGTCCACCTTCCAGCAGATGT & AGCTCAGTAACAGTCCGCCTAGA \\
\hline HK2 & TTTCACCTTCTCGTTCCCCT & GTCATTCACCACAGCCACAA \\
\hline PDK4 & CCTTCACACCTCACCACAT & AAAGAGGCGGTCAGTAATCC \\
\hline GLUT 1 & TCAACACGGCCTTCACTG & CACGATGCTCAGATAGGACATC \\
\hline LDHA & GGACAGTGCCTACGAGGTGAT & GGATGCACCCGCCTAAGG \\
\hline LDHB & GGGAAAGTCTCTGGCTGATGAA & CTGTCACAGAGTAATCTTTATCGGC \\
\hline DC-STAMP & AAAACCCTTGGGCTGTTCTT & AATCATGGACGACTCCTTGG \\
\hline OSCAR & TCGCTGATACTCCAGCTGTC & ATCCCAGGAGTCACAACTGC \\
\hline CATH.K & ATATGTGGGCCAGGATGAAAGTT & TCGTTCCCCACAGGAATCTCT \\
\hline
\end{tabular}

Extracellular flux assay. Real time bioenergetic profile of OCPs were obtained by measuring oxygen consumption rate (OCR) and extracellular acidification rate (ECAR) using the XFe96 extracellular flux analyzer (Seahorse Bioscience, Agilent Technologies, North Billerica, MA). Briefly, OCPs were seeded at a density of 150.000 cells per well (96-well-plate) and left untreated or treated with RANKL $(10 \mathrm{ng} / \mathrm{ml})$ in growth medium with and without bone powder for $48 \mathrm{~h}$. On the day of measurement, cells were washed two times with Seahorse XF Base Medium and.

growth medium was replaced by Seahorse XF Base Medium and supplemented with glucose $(10 \mathrm{mM})$, sodium-pyruvate $(200 \mathrm{mM})$ and glutamax $(200 \mathrm{mM})$. Further, medium was warmed up to $37^{\circ} \mathrm{C}$ and $\mathrm{pH}$ was subsequently adjusted to $7.4 \pm 0.1$. For Glyco Stress assay, no glucose and pyruvat was added to Seahorse XF Basismedium. Following incubation in an incubator without $\mathrm{CO}_{2}$ at $37^{\circ} \mathrm{C}$ for $60 \mathrm{~min}$, basal OCR and ECAR were recorded for $105 \mathrm{~min}$. Mito Stress assay was performed by sequential addition of $20 \mu \mathrm{M}$ oligomycin (inhibitor of ATP synthesis), $10 \mu \mathrm{M}$ carbonyl cyanide 4-(trifluoromethoxy) phenylhydrazone (FCCP, uncoupling agent) and $10 \mu \mathrm{M}$ rotenone/antimycin A (inhibitors of complex I and complex III of the respiratory chain, respectively). Glyco Stress assay was performed by sequential addition of $100 \mathrm{mM}$ glucose, $20 \mu \mathrm{M}$ oligomycin and $500 \mathrm{mM}$ 2dG. Parameters such as ATP-linked OCR, maximal OCR, spare respiratory capacity (SRC) and glycolysis were evaluated using the Wave XFe Analyzer software Wave Desktop 2.6 (PC only) and Wave Controller 2.6 (https ://www.agilent.com/).

Glucose and lactate measurements. Glucose and lactate content were determined as $\mathrm{mg} / \mathrm{dl}$ in cell culture supernatants using a SuperGLcompact (Hitado, Möhnesee, Germany) according to the manufacturers standard operating procedure.

Measurement of glycolysis and Krebs cycle metabolites. Phosphorylated intermediates and carboxylates were extracted with perchloric acid from $\mathrm{xx}$ to $\mathrm{xx}$ mg samples of shock-frozen $\mathrm{xxx}$ tissue for four biological replicates as described previously ${ }^{13}$ applying ionchromatography with an ICS3000 HPLC- system (Dionex) and ESI/MS/MS detection using a QTrap3200 Triple-Quadrupole massspectrometer with turbo V ion source (Applied Biosystems) operated in multiple reaction monitoring mode.

Cytotoxicity assay. Cytotoxicity assay was performed with CytoTox 96 Non-Radioactive Cytotoxicity Assay from Promega as a measurement of lactate dehydrogenase (LDH) release into the supernatant according to the manufactors protocol. During experimental treatment via bone resorption assay, supernatant samples were cleared from non adherent cells and transferred to a 96 plate and an equal volume of CytoTox 96 reagent is added to each well and incubated for $30 \mathrm{~min}$. Stop solution is added and the absorbance signal is measured at $490 \mathrm{~nm}$ in a plate reader. Results were calculated by subtracting the average values of the culture medium background from all values of experimental wells to compute percent cytotoxicity.

Statistical analysis. Statistical analyses were performed using GraphPad Prism 8 software (https:// www.graphpad.com/scientific-software/prism/). Results are depicted as median \pm interquartile range (IQR) if not stated otherwise. For a two-group comparison, a Student's t-test was applied if the pretest for normality (D’Agostino-Pearson normality test) was not rejected at the 0.05 significance level; otherwise, a Mann-Whitney U-test for nonparametric data was used. $P$ values less than 0.05 were considered significant. Results are expressed as mean \pm SE (SEM). No statistical method was used to predetermine sample size.

Received: 11 September 2020; Accepted: 17 November 2020

Published online: 03 December 2020 


\section{References}

1. Teitelbaum, S. L. \& Ross, F. P. Genetic regulation of osteoclast development and function. Nat. Rev. Genet. 4(8), 638-649 (2003).

2. Xing, L., Xiu, Y. \& Boyce, B. F. Osteoclast fusion and regulation by RANKL-dependent and independent factors. World J. Orthop. 3(12), 212-222 (2012).

3. Teitelbaum, S. L. Bone resorption by osteoclasts. Science 289(5484), 1504-1508 (2000).

4. Kim, J. M. et al. Osteoclast precursors display dynamic metabolic shifts toward accelerated glucose metabolism at an early stage of RANKL-stimulated osteoclast differentiation. Cell. Physiol. Biochem. 20(6), 935-946 (2007).

5. Indo, Y. et al. Metabolic regulation of osteoclast differentiation and function. J. Bone Miner. Res. 28(11), 2392-2399 (2013).

6. Arnett, T. R. \& Orriss, I. R. Metabolic properties of the osteoclast. Bone 115, 25-30 (2018).

7. Lemma, S. et al. Energy metabolism in osteoclast formation and activity. Int. J. Biochem. Cell Biol. 79, 168-180 (2016).

8. Van den Bossche, J., O’Neill, L. A. \& Menon, D. Macrophage immunometabolism: where are we (going)?. Trends Immunol. 38(6), 395-406 (2017).

9. Komori, T. Animal models for osteoporosis. Eur. J. Pharmacol. 759, 287-294 (2015).

10. Regan, J. N. et al. Up-regulation of glycolytic metabolism is required for HIFlalpha-driven bone formation. Proc. Natl. Acad. Sci. U. S. A. 111(23), 8673-8678 (2014).

11. Müller, D. I. H. et al. PPAR $\delta$-mediated mitochondrial rewiring of osteoblasts determines bone mass. Sci. Rep. 10(1), 8428 (2020).

12. Kronke, G. et al. Oxidized phospholipids induce expression of human heme oxygenase-1 involving activation of cAMP-responsive element-binding protein. J. Biol. Chem. 278(51), 51006-51014 (2003).

13. Hofmann, J. et al. Detecting functional groups of Arabidopsis mutants by metabolic profiling and evaluation of pleiotropic responses. Front. Plant. Sci. 2, 82. https://doi.org/10.3389/fpls.2011.00082 (2011).

\section{Acknowledgements}

The present work was performed in fulfilment of the requirements for obtaining the degree "dr. med." We thank Dana Weidner for excellent technical assistance. This work was supported by the IZKF Erlangen (J.T.), the Deutsche Forschungsgemeinschaft (FG 2886 "PANDORA" to G.K., D.M. and G.S. as well as the CRC1181 to G.K. and G.S). Additional support was provided by the Emerging Field Initiative (EFI) of the Friedrich-Alexander University Erlangen-Nürnberg (FAU) and the STAEDTLER Stiftung (EFI_Verbund_Med_05_MIRACLE to G.K., U.S. and J.H.) and the Bundesministerium für Bildung und Forschung (BMBF) (METARTHROS to G.K. and G.S.) and the European Union (Horizon 2020 ERC-2014-StG 640087 - SOS to G.K and Horizon 2020 ERC-2018SyG nanoSCOPE as well as RTCure to G.S.).

\section{Author contributions}

J.T. designed the study, performed and interpreted experiments. C.St., C.B., J.T., B.K., S.A, D.I.H.M and M.F. performed experiments, collected, and interpreted data. D.M. and M.B. established and performed the metabolic characterization of osteoclasts. U.S and J.H performed metabolic analysis. J.T., G.S., C.S. and G.K. designed the study and experiments and wrote the manuscript. All authors read and commented on the manuscript.

\section{Competing interests}

The authors declare no competing interests.

\section{Additional information}

Supplementary information is available for this paper at https://doi.org/10.1038/s41598-020-77892-4.

Correspondence and requests for materials should be addressed to G.K. or C.S.

Reprints and permissions information is available at www.nature.com/reprints.

Publisher's note Springer Nature remains neutral with regard to jurisdictional claims in published maps and institutional affiliations.

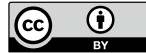

Open Access This article is licensed under a Creative Commons Attribution 4.0 International License, which permits use, sharing, adaptation, distribution and reproduction in any medium or format, as long as you give appropriate credit to the original author(s) and the source, provide a link to the Creative Commons licence, and indicate if changes were made. The images or other third party material in this article are included in the article's Creative Commons licence, unless indicated otherwise in a credit line to the material. If material is not included in the article's Creative Commons licence and your intended use is not permitted by statutory regulation or exceeds the permitted use, you will need to obtain permission directly from the copyright holder. To view a copy of this licence, visit http://creativecommons.org/licenses/by/4.0/.

(C) The Author(s) 2020 